\title{
Diesel Aerosol Sampling in the Atmosphere
}

David Kittelson, Jason Johnson, Winthrop Watts, Qiang Wei, Marcus Drayton and Dwane Paulsen

Center for Diesel Research, University of Minnesota

Nicolas Bukowiecki

Paul Scherrer Institute 
SAE routinely stocks printed papers for a period of three years following date of publication. Direct your orders to SAE Customer Sales and Satisfaction Department.

Quantity reprint rates can be obtained from the Customer Sales and Satisfaction Department.

To request permission to reprint a technical paper or permission to use copyrighted SAE publications in other works, contact the SAE Publications Group.

This article was prepared as an account of work sponsored by an agency of the United States

Government. Neither the United States Government nor any agency thereof, nor any of their employees, makes any warranty, express or implied, or assumes any legal liability or responsibility for the accuracy, completeness, or usefulness of any information, apparatus, product, or process disclosed, or represents that its use would not infringe privately owned rights. Reference herein to any specific commercial product, process, or service by trade name, trademark, manufacturer, or otherwise does not necessarily constitute or imply its endorsement, recommendation, or favoring by the United States Government or any agency thereof. The views and opinions of authors expressed herein do not necessarily state or reflect those of the United States Government or any agency thereof.

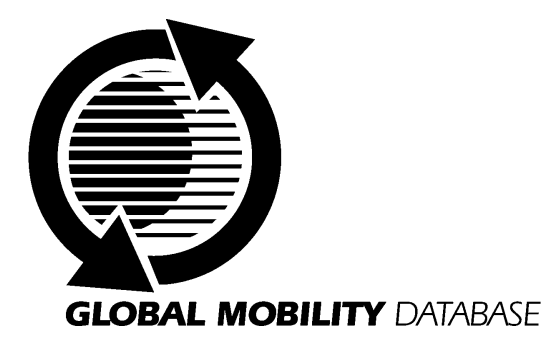

All SAE papers, standards, and selected books are abstracted and indexed in the Global Mobility Database

ISSN 0148-7191

No copyright is asserted in the works of U.S. Government employees.

Positions and opinions advanced in this paper are those of the author(s) and not necessarily those of SAE. The author is solely responsible for the content of the paper. A process is available by which discussions will be printed with the paper if it is published in SAE Transactions. For permission to publish this paper in full or in part, contact the SAE Publications Group.

Persons wishing to submit papers to be considered for presentation or publication through SAE should send the manuscript or a 300 word abstract of a proposed manuscript to: Secretary, Engineering Meetings Board, SAE.

\section{Printed in USA}




\title{
Diesel Aerosol Sampling in the Atmosphere
}

\author{
David Kittelson, Jason Johnson, Winthrop Watts, \\ Qiang Wei, Marcus Drayton and Dwane Paulsen \\ Center for Diesel Research, University of Minnesota
}

Nicolas Bukowiecki

Paul Scherrer Institute

No copyright is asserted in the works of U.S. Government employees

\begin{abstract}
The University of Minnesota Center for Diesel Research along with a research team including Caterpillar, Cummins, Carnegie Mellon University, West Virginia University (WVU), Paul Scherrer Institute in Switzerland, and Tampere University in Finland have performed measurements of Diesel exhaust particle size distributions under real-world dilution conditions. A mobile aerosol emission laboratory (MEL) equipped to measure particle size distributions, number concentrations, surface area concentrations, particle bound PAHs, as well as $\mathrm{CO}_{2}$ and $\mathrm{NO}_{\mathrm{x}}$ concentrations in real time was built and will be described. The MEL was used to follow two different Cummins powered tractors, one with an older engine (L10) and one with a state-of-the-art engine (ISM), on rural highways and measure particles in their exhaust plumes. This paper will describe the goals and objectives of the study and will describe representative particle size distributions observed in roadway experiments with the truck powered by the ISM engine.
\end{abstract}

\section{INTRODUCTION}

Recent studies have linked environmental exposure to fine particles less than $2.5 \mu \mathrm{m}$ aerodynamic diameter to adverse health effects [1-6], although no causal mechanisms have been identified. The relationship between fine particles and health is a logical link because the efficiency of particle deposition in the respiratory tract is a function of particle size. Figure 1 illustrates relationships between idealized Diesel particulate matter (DPM) number and mass weighted size distributions [7] and the alveolar deposition curve $[8,9]$. As DPM increases in size, the deposition efficiency decreases.

DPM follows a lognormal, trimodal size distribution with the concentration in any size range being proportional to the area under the corresponding curve in that range
[10-15]. Nuclei-mode particles range in diameter from 0.005 to $0.05 \mu \mathrm{m}(5-50 \mathrm{~nm})$. Based on physical arguments, they are believed to consist of metallic compounds, elemental carbon and semi-volatile organic and sulfur compounds that form particles during exhaust dilution and cooling $[13,14,15]$. The nuclei mode typically contains $1-20 \%$ of the particle mass and more than $90 \%$ of the particle number. The accumulation mode ranges in size from roughly 0.05 to $0.5 \mu \mathrm{m}(50-500 \mathrm{~nm})$. Most of the mass, composed primarily of carbonaceous agglomerates and adsorbed materials, is found here. The coarse mode consists of particles larger than $1 \mu \mathrm{m}$ and contains $5-20 \%$ of the DPM mass. These relatively large particles are formed by reentrainment of particulate matter, which has been deposited on cylinder, and exhaust system surfaces. Also shown in Figure 1 are size range definitions for atmospheric particles: PM10 (diameter < $10 \mu \mathrm{m}$ ), fine particles (diameter $<2.5 \mu \mathrm{m}$ ), nanoparticles (diameter $<0.05 \mu \mathrm{m}$ or $<50 \mathrm{~nm}$ ), and ultrafine particles (diameter $<0.10 \mu \mathrm{m}$ or $<100 \mathrm{~nm}$ ).

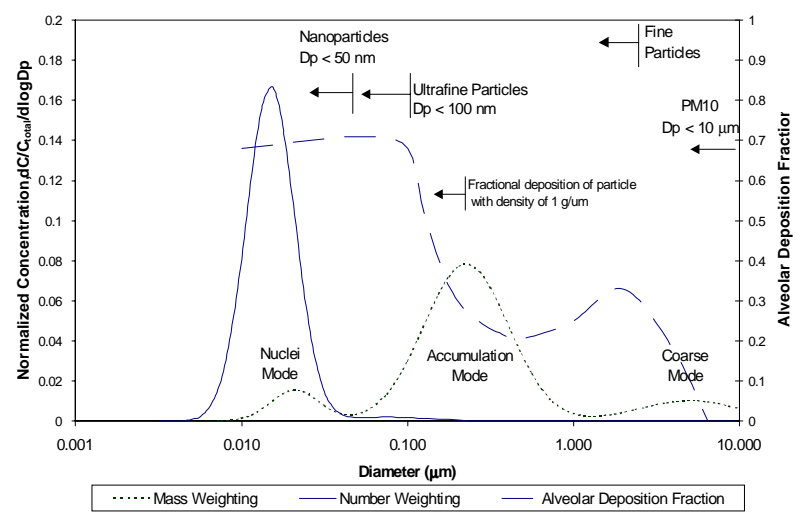

Figure 1. Typical Engine Exhaust Mass and Number Weighted Size Distributions Shown with Alveolar Deposition Fraction 


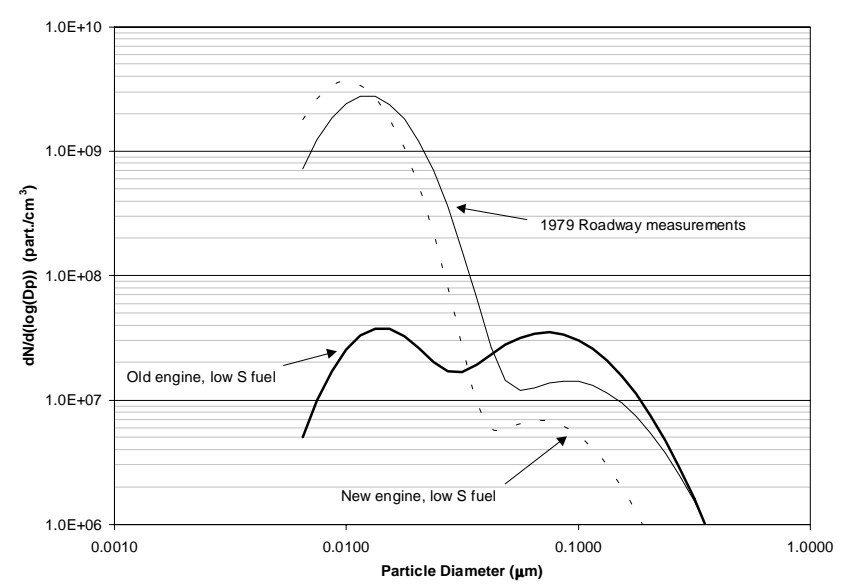

Figure 2 - Number Size Distribution Data from HEI Report (Bagley et al. [16]) and 1979 CRC Roadway Study (Kittelson et al. [22])

The interest in nanoparticle emissions from internal combustion engines, particularly Diesel, has been recently heightened by engine laboratory studies that showed an increase in nanoparticle emissions from low-mass emission engines, and engines equipped with emission control technologies such as oxidation catalysts and/or traps [16-19]. In a study funded by the Health Effects Institute (HEI), Bagley et al. [16] compared size distributions from a 1988 Cummins engine with those from a 1991 engine of the same family. Figure 2 includes two plots of the number concentration distribution function, $d N / \operatorname{dlog}\left(D_{p}\right)$, against particle diameter, $D_{p}$, that have been constructed from data given in that study. Compared to the 1988 engine, the 1991 engine showed a roughly 3 fold decrease in mass emissions (indicated by the decrease in $\mathrm{dN} / \mathrm{d} \log \left(D_{\mathrm{p}}\right)$ in the accumulation mode size range) but a 10 to 30 fold increase in number concentration (indicated by the $d N / d \log \left(D_{p}\right)$ increase in the nuclei mode size range). This raised concerns that new, low mass emission engines might be producing a new problem in high emissions of nanoparticles. Although this concern about nanoparticle emissions is new, nanoparticle emissions are not. High concentrations of nanoparticles have been observed on and near roadways for many years [20-24]. Data from a 1979 roadway study [22] are also shown in Figure 2. These data were collected with a mobile laboratory driving on a rural freeway behind a tractor-trailer powered by Cummins engine from the same engine family as those used in the HEI study. The results are similar to those observed for the 1991 engine used in the HEI study.

The basic question is whether nanoparticle emissions from engines are changing as technology improves and emission standards are made more stringent. It is an objective of the project described here to answer this question. Often more than $90 \%$ of the nanoparticles emitted by engines are formed from volatile particle precursors during exhaust dilution [25-27]. These precursors are presumably the lower vapor pressure compounds usually associated with Diesel particulate matter, like sulfuric acid and condensable hydrocarbons. Particle dynamics during sampling and dilution are highly nonlinear - large changes in particle number may result from small changes in dilution and sampling conditions. Sampling and dilution parameters like dilution ratio, temperature, humidity, and residence time strongly influence nanoparticle formation. Up to two orders of magnitude difference in nanoparticle emissions were observed for an engine running at the same steady-state condition, but with different dilution schemes [26] as illustrated in Figure 3. Similar sensitivity was observed to primary dilution ratio and primary dilution temperature [26].

Nucleation and growth of particle precursors to form nanoparticles during dilution are strongly influenced by the presence of other particles [27]. When carbon is removed from the exhaust, material that would adsorb onto carbonaceous agglomerates nucleates and grows to form nanoparticles. This process is very nonlinear and strongly dependent upon dilution conditions. Thus, the influence of dilution conditions may be even greater when most of the solid carbon is removed from the exhaust. Changes up to nearly four orders of magnitude in nanoparticle concentration with changing dilution conditions were observed when the same engine as used in [26] was fitted with a wall-flow exhaust particle filter [25].

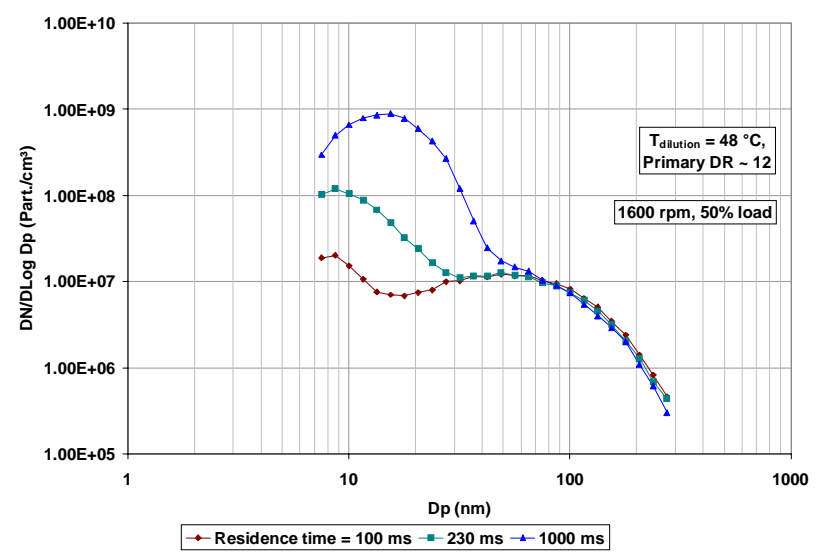

Figure 3. Typical Weighted Size Distributions from Abdul-Khalek, et al., [26] Showing the Influence of Residence Time in the First Stage of a Two-Stage Dilution System. Medium-Duty Diesel Engine Running and Medium Speed and Load.

As engines become cleaner, it will become increasingly difficult to make representative measurements of exhaust size distributions. The Coordinating Research Council (CRC) E-43 program, described here, is intended to determine how to make such measurements. This paper is a progress report on the E-43 program, which is currently only about $25 \%$ complete. Expect that future information derived from this research program will recommend which combination of primary dilution ratio, residence time, primary dilution temperature, and other factors will best represent real world dilution conditions. 


\section{OBJECTIVES OF THE CRC E-43 PROGRAM}

There are four primary objectives of the CRC E-43 Program:

1. Measure on-road particle size distributions (number, volume, surface area) in the exhaust plume of heavyduty Diesel vehicles.

2. Attempt to reproduce these results under laboratory conditions (chassis dynamometer, wind tunnel, engine dynamometer).

3. Model atmospheric aging and dispersion of freshly emitted Diesel aerosols.

4. Determine size fractionated chemical composition of Diesel particulate matter collected in the laboratory and in a wind tunnel.

These objectives are described in more detail in [28]. A very important fundamental goal of the project is to understand the nature of the tailpipe to nose process as it takes place on and near roadways. This is where the highest exposures to nanoparticles are likely to occur.

The project may be divided into 7 phases:

1. On-road chase experiments with an old technology and a new technology Cummins powered tractors,

2. Wind tunnel tests with the new technology Cummins powered tractor,

3. Chassis dynamometer experiments with the two Cummins powered tractors,

4. Engine dynamometer tests with the engine from the new technology Cummins powered tractor,

5. On-road chase experiments with an old technology and a new technology Caterpillar powered tractors,

6. Chassis dynamometer experiments with the two Caterpillar powered tractors,

7. Engine dynamometer tests with several new technology Caterpillar engines.

The experimental portions of phases 1 and 2 have been completed. A very large body of data has been collected and partially analyzed. This paper will describe some of the initial results from phase 1 of the project.

\section{EXPERIMENTAL PROCEDURE AND RESULTS}

The heart of the project is the mobile emission laboratory (MEL). It has been designed and built to allow comparison of Diesel exhaust aerosol characteristics measured in chase experiments under real world highway conditions with simulations of those conditions in a wind tunnel, on chassis dynamometers, and on engine dynamometers. The MEL was installed in a cargo container that was mounted on a Volvo container truck. During the chase experiments, illustrated in Figure 4, exhaust plumes are collected with a $3.6 \mathrm{~m}$ long aluminum boom mounted over the cab of the truck. The boom brings the sample stream to a distribution manifold in the interior of the MEL. A flow-regulating vane pump provides a large bypass flow for the instruments maintains constant suction at the inlet of the boom. The primary particle-sizing instrument used in these experiments is a TSI scanning mobility particle sizer (SMPS). It was used to obtain particle size distributions in the 9 to $300 \mathrm{~nm}$ diameter range. This instrument requires at least $30 \mathrm{~s}$ to complete an entire size scan and is not suitable for making measurements in the rapidly varying plume encountered in chase experiments. Therefore it was necessary to use a bag sampler with the SMPS. A portion of the aerosol entering the sampling boom is used to fill a large plastic bag in about $5 \mathrm{~s}$ to obtain a snapshot of the plume for analysis by the SMPS. One or more size distribution scans are run on each bag sample, each taking about 90 s. Repeated scans have shown that the bag sample procedure does not significantly change the size distribution of the collected aerosol. Furthermore, comparisons between direct samples (bypassing the bag) and bag samples obtained with steady aerosols show no significant differences. On the other hand, all size distributions reported here are for fresh roadway aerosols with little atmospheric aging. The role of aging is being addressed in modeling effort that is a part of the E-43 program, but has not yet been completed.

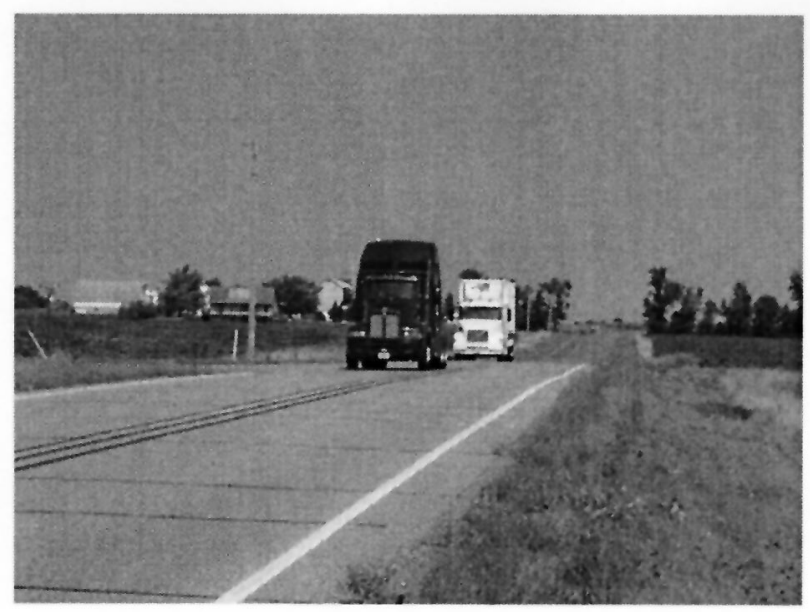

Figure 4. University of Minnesota, E-43, Mobile Aerosol Laboratory during a Roadway Chase Experiment.

The other aerosol and gas instruments in the MEL sampled continuously from the distribution manifold. The instruments used in the Cummins chase experiments included the following:

1. An electrostatic low pressure impactor (ELPI) to determine particle size distributions based on the aerodynamic aerosol diameter in the 29 to $2500 \mathrm{~nm}$ range.

2. A condensation particle counter (CPC) to measure total aerosol number concentrations for particles larger than about $3 \mathrm{~nm}$. 
3. An epiphaniometer to determine the Fuchs surface area of the aerosol [29]. This well characterized measuring method is the standard for the other surface measuring instruments, despite its relatively slow (5 minute) time resolution.

4. A photoelectric aerosol sensor (PAS) to measure photoemitting substances on the surface of aerosol particles. Diesel soot has been found to show a large response.

5. A diffusion charger (DC), consisting of a diffusion charging section combined with a current sensor, to measure the surface area concentration of the aero$\mathrm{sol}$. Its fast response time $(0.5 \mathrm{~s})$ made the instrument useful as a fast plume indicator. The combination DC/PAS has been described as a powerful tool for the identification of Diesel soot [30].

6. High sensitivity $\mathrm{CO}_{2}$ and $\mathrm{NO}_{\mathrm{x}}$ analyzers to track the plume and in conjunction with a stack sampler mounted in the chased truck used to determine dilution ratios.

The MEL was used during on-highway, truck chase experiments. The following test conditions were used in the Cummins chase experiments: idle; $55 \mathrm{mph}$ cruise, light and heavy load; and $40-55 \mathrm{mph}$ acceleration, light and heavy load. We also attempted to do chase experiments during decelerations but the plumes were too weak to detect. Two different tractors were used, a 1999 model powered by a 1999 specification ISM engine and a 1988 model powered by a 1988 L10 engine. Two fuels were used with the ISM engine: a standard federal fuel and a reformulated California fuel. Only the federal fuel was run in the L10 tests. The Diesel exhaust plume of the truck being chased was captured and analyzed 1-3 s after it was emitted from the exhaust stack. Following distances ranged from about 15 to $50 \mathrm{~m}$. The plume was tracked by monitoring the DC and/or NO signal in real time. When a strong steady signal was observed, a bag sample was taken.

SMPS SIZE DISTRIBUTION MEASUREMENTS - Results are reported here for the 1999 ISM engine running on the California fuel under light load cruise and acceleration conditions. Figures 5 and 6 show results of SMPS scans on bag samples obtained in replicate chase experiments for cruise and acceleration conditions. Plume and background samples are shown. These are uncorrected measurements so that the absolute concentrations are expected to vary with the extent of dilution of the plume at the time the sample is obtained. The size distribution shape similarities of Figures 5 and 6 indicate that roadway experiments were replicated well even though a roadway test is more difficult to control than a laboratory test. Each data curve in these two figures represents slightly different dilution because roadway conditions are not exactly the same. Although the absolute concentrations go up and down with dilution, the shapes are quite consistent for a given operating and sampling condition.
The plume concentrations may be converted to stack equivalent stack concentrations using the following relationship:

$$
\left.\frac{d N}{d \log \left(D_{p}\right)_{\text {stack }}}=\left(\frac{d N}{d \log \left(D_{p}\right)}\right)_{\text {plume }}-\frac{d N}{d \log \left(D_{p}\right)_{\text {background }}}\right) \times \text { Dilution Ratio }
$$

Dilution ratios are being determined from stack, plume, and background NO concentrations. However, validations of these values are still underway. Regardless of whether or not the dilution ratio is known, the shape of the size distribution is determined from the plume minus background difference distributions. These difference distributions may be conveniently compared by normalizing them to a unit volume concentration of $1 \mu \mathrm{m}^{3} / \mathrm{cm}^{3}$. When a size distribution is normalized in this manner it has the convenient property of giving the number emitted per unit mass emitted because it is reasonable to assume that the volume and mass concentrations are proportional to one another [15]. Figure 7 shows such normalized distributions for data obtained on two consecutive days. The shapes of these size distributions are quite similar except that the data for the cooler day suggest relatively more nuclei mode particles are formed during dilution under cooler ambient conditions. This is not surprising because the vapor pressures of condensable species like sulfuric acid and high molecular weight hydrocarbons are lower at lower temperatures so that the driving force for nucleation and growth of nanoparticles will be stronger. This is consistent with the common observation of steam plumes behind vehicles in cold winter weather.

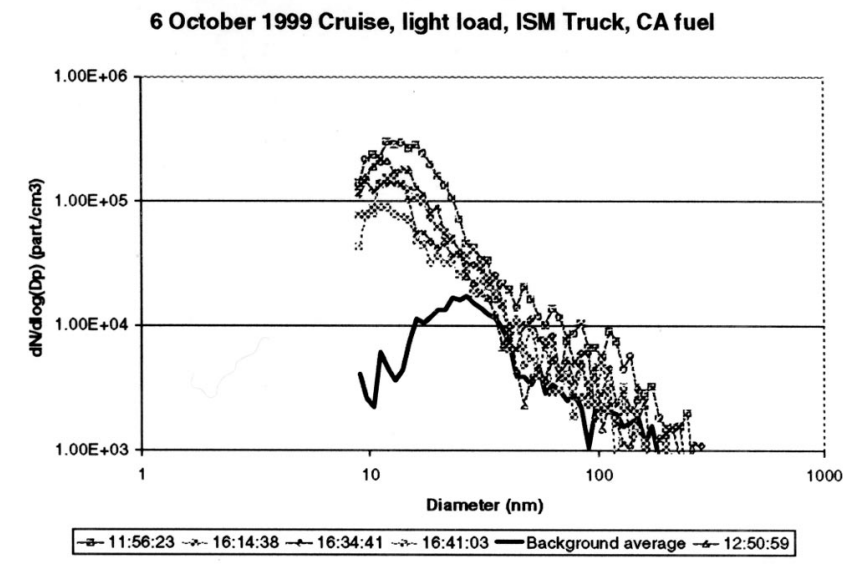

Figure 5. Typical Chase Experiment Size Distributions. California Fuel, Light Load, $55 \mathrm{mph}$ Cruise with ISM Engine Powered Truck.

We have also observed an increase in nanoparticle formation during dilution under laboratory conditions intended to simulate atmospheric dilution. Figure 8 shows this effect using a single stage dilution tunnel developed to simulate atmospheric dilution temperatures and rates by Wei [31]. These experiments were done 
with a 1995 medium-duty Diesel engine designed to meet 1994 heavy-duty standards running at medium speed and load. Note the effect of dilution temperature on nanoparticle formation. The concentration of particles in the nuclei mode increases by nearly a factor of 10 as the temperature is reduced from 25 to $15 \mathrm{C}$. This suggests that there could be differences in the tendency to form volatile nanoparticles between, for example, Minnesota and Arizona, not because of changes in engine emissions but because of changes in what happens in the atmosphere.

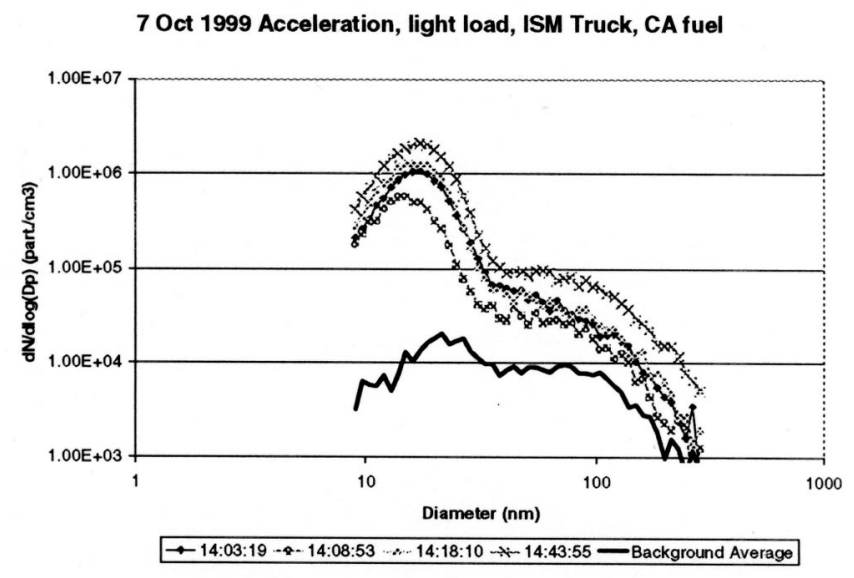

Figure 6. Typical Chase Experiment Size Distributions. California Fuel, Light Load, 40-45 mph Acceleration with ISM Engine Powered Truck.

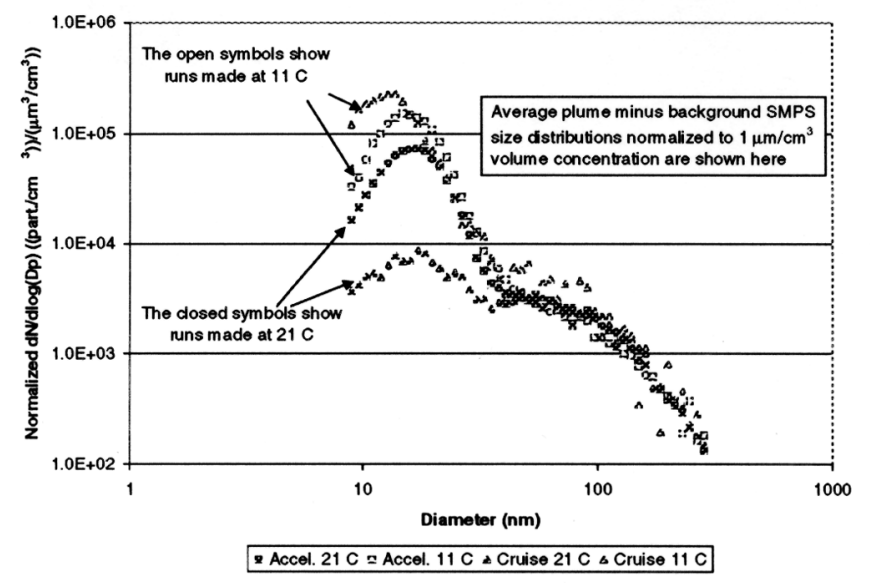

Figure 7. Normalized Average Difference Size Distributions for 2 Consecutive Days, 6 and 7 October 1999 with Different Ambient Temperatures. The Results Are Normalized to a Unit Volume Concentration of $1 \mu \mathrm{m}^{3} / \mathrm{cm}^{3}$. California Fuel, ISM Truck, Light Load.

In addition to the temperature effect observed here is the important consideration of how long these nanoparticles will survive in what atmospheres, under what dilution conditions, and if, after reaching a decision of how to most appropriately dilute, how important this temperature effect will be at that dilution condition. At present, the time dimension (i.e., the lifetime) of these nanoparticles has not been determined, and is planned as future research.

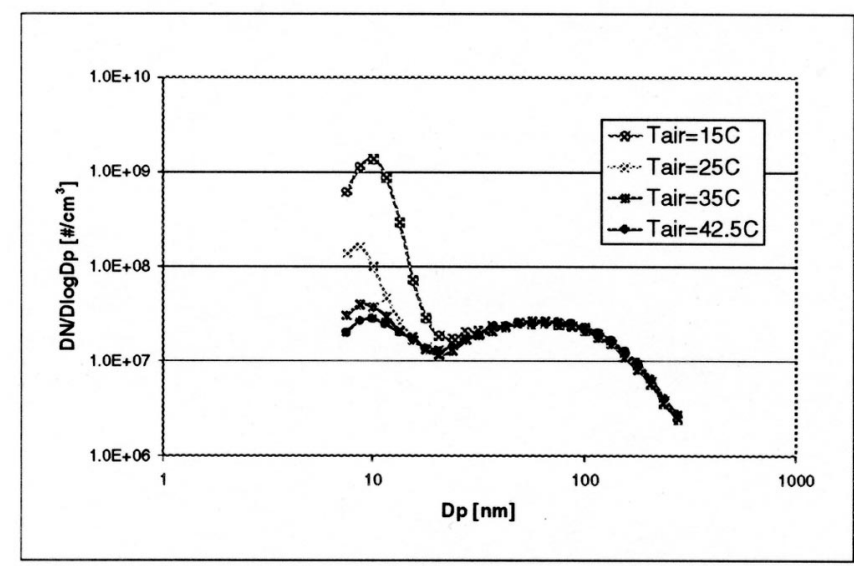

Figure 8. Influence of Ambient Dilution Temperature Using a Single Stage Dilution Tunnel Designed to Simulate the Rate, Temperature, and Extent of Dilution Under Atmospheric Conditions. The Tunnel is Operated to Give a Dilution Ratio of 1000, 1 s Downstream of Sample Introduction.

MEASUREMENTS MADE WITH REAL TIME PARTICLE INSTRUMENTS - The Cummins chase experiments have yielded more than 400,000 individual records and we have only started to analyze this vast data set. However to give a sense for the type of data collected some representative $\mathrm{DC}$ and $\mathrm{CPC}$ data are shown below. It is necessary to correct for differences in lag and response times when comparing continuous instruments. Their response lag times were determined by repeatedly switching between a sample stream and a filtered stream. It was found that the combined internal and transfer line lag time of the CPC was $6 \mathrm{~s}$ longer than that of the DC. Therefore in the data shown below the CPC response is shifted $6 \mathrm{~s}$ relative to the DC response. Figure 9 shows the instantaneous variations of the $D C$ and $\mathrm{CPC}$ response during a short time window of the chase experiments for the ISM truck, 55-mph cruise, light load, California fuel. Note that the instruments track very well. Figure 10 is a plot of the DC response against the CPC response for a more extended time period, but the same operating conditions. A linear regression line through the data is also shown and has an $r^{2}$ value of 0.75 . Assuming that the background and stack conditions are steady, the slope of such a plot is the ratio of the particle surface to the particle number added by the plume, or the $\mathrm{S} / \mathrm{N}$ ratio. This ratio may be related to the diameter of average surface, $D_{\text {as }}$, through:

$$
D_{a s}=\left(S / \pi_{N}\right)^{0.5}
$$

For the data shown in Figure 9, $D_{a s}$ is $25 \mathrm{~nm}$. 


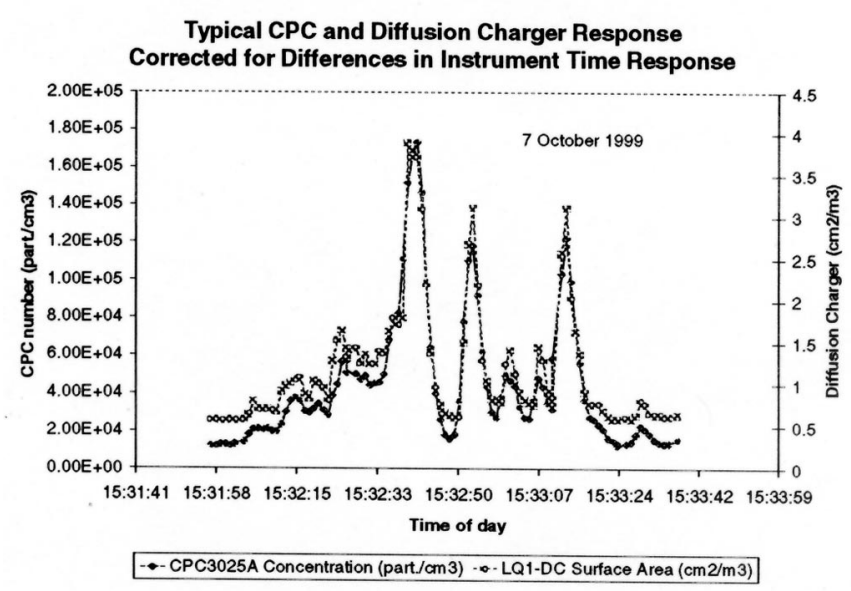

Figure 9. Comparison between Condensation Particle Counter and Diffusion Charger Response for a Short, but Representative Time Window during Roadway Chase Experiments on 7 October 1999. ISM Powered Truck, CA Fuel, 55 mph Cruise, Light Load.

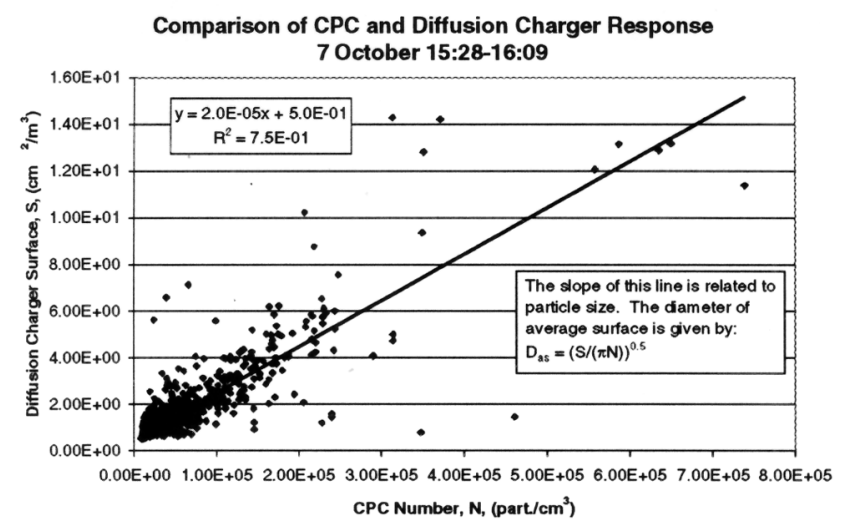

Figure 10. Comparision between Condensation Particle Counter and Diffusion Charger Response for a Time Window during Roadway Chase Experiments on 7 October 1999. ISM Powered Truck, CA Fuel, 55 mph Cruise, Light Load.

\section{CONCLUSIONS AND ONGOING WORK}

The experimental work for the first 2 phases of the CRC E-43 Project, Cummins chase experiments and wind tunnel experiments have been completed, but analysis of the collected data continues. Some preliminary results of the chase experiments have been reported here.

The mobile emission laboratory built for this project has performed very well in the Cummins chase experiments. These experiments have shown that it is feasible to characterize the particle emissions of Diesel powered vehicles under real world conditions. For the engine and test conditions that we have encountered the sensitivity of nanoparticle formation to dilution temperature suggests that these nanoparticles are volatile and form by nucle- ation during dilution. Nucleation is highly nonlinear and very sensitive to conditions. Consequently the first step in understanding this process, and in accessing the nature of real world exposures, must be taken under real world conditions. We have found that for the engines and conditions examined so far, the SMPS size distributions are quite similar in shape to those found in some laboratory studies. The submicron size distributions are bimodal (the supermicron coarse particle mode is not considered here) with a nuclei mode in the 10 to $20 \mathrm{~nm}$ range containing 60 to $95 \%$ of the number and an accumulation mode in the 40 to $60 \mathrm{~nm}$ range containing 90 to $99 \%$ of the volume (or mass). The main chase experiment sampling parameter observed to influence the size distribution is ambient temperature, with larger relative concentrations of nanoparticles formed during dilution at lower ambient temperatures.

Ongoing work includes instrument and sampling system calibration and validation as part of a quality assurance program. We expect to do chassis and engine dynamometer tests at Cummins in April and May 2000; chase, chassis and engine dynamometer experiments with Caterpillar engines during the late spring and summer of 2000.

In addition to these general test descriptions and locations are the more complex aerosol physics concepts that need to be resolved. More on-road particle size distributions will be obtained to verify data collected thus far. The Cummins and Caterpillar tests will compare laboratory dilution tunnel size distributions and concentrations with on-highway data to determine the proper laboratory method of dilution rate, ratio and aerosol residence time. Atmospheric aging and dispersion of freshly emitted Diesel aerosols will be modeled for comparison with data obtained in these experiments. Finally, although the low mass of nanoparticles makes chemical analysis challenging, a limited number of chemical analyses are planned.

\section{ACKNOWLEDGEMENTS}

This work is part of the CRC E-43 Project, "Diesel Aerosol Sampling Methodology" Prime Contractor: University of Minnesota. Subcontractors: West Virginia University, Paul Scherrer Institute, Carnegie Mellon University, Tampere University. Sponsors: Coordinating Research Council and the National Renewable Energy Laboratory with co-sponsorship from the Engine Manufacturers Association, the SouthCoast Air Quality Management District, the California Air Resources Board, Cummins, Caterpillar, and Volvo. Background information is based on work sponsored by Perkins Engine Company

We would also like to thank the students who have helped to build and test the MEL and to take part in the experiments. They include Megan Arnold, Feng Cao, Erin Ische, Heejung Jung, and Jungwoo Ryu. 


\section{REFERENCES}

1. Dockery, D.W., Pope, C.A., Xu, X., Spengler, J.D., Ware, J.H., Fay, M.E., Ferris, B.G., and Speizer, F.E., "An Association Between Air Pollution and Mortality in Six U.S. Cities", Massachusetts Medical Society Journal of Medicine, Volume 329, Number 24, pp 1753-1759 (1993).

2. Donaldson, K., Beswick, P.H., and Gilmour, P.S., "Free Radical Activity Associated with the Surface of Particles: a Unifying Factor in Determining Biological Activity?", Toxicology Letters, Volume 88, pp 293-298 (1996).

3. Ferin, J., Oberdorster, G., and Penney, D.P., "Pulmonary Retention of Ultrafine and Fine Particles in Rats", American Journal of Respiratory Cell Molecular Biology, Volume 6, pp 535-542 (1992).

4. McAughey, J. J., "Regional Lung Deposition and Dose of Ambient Particulate in Humans by Particle Mass and Number", Research Report, AEA Technology, Aerosol Science Centre, Oxfordshire, UK (1997).

5. Pope, C.A., Thun, M.J., Namboodriri, M.M., Dockery, D.W., Evans, J.S., Speizer, F.E., and Heath, C.W., "Particulate Air Pollution as a Predictor of Mortality in a Prospective Study of U.S. Adults", American Journal of Respiratory Critical Care Medicine, Volume 151, pp 669-674 (1995).

6. Seaton, A., W. MacNee, K. Donaldson, D. Godden. 1995. Particulate Air Pollution and Acute Health Effects. The Lancet, Vol. 345, excerpt from section on -- How particles may cause harm, p. 177.

7. Whitby, K. T. and B. K. Cantrell. 1975. Atmospheric Aerosols - Characteristics and Measurement. ICESA Conference Proceedings, IEEE \#75-CH 1004-1 ICESA, paper 29-1, $6 \mathrm{pp}$.

8. Morrow, P. E., D. V. Bates, B. R. Fisher, et al. 1964. Deposition and Retention Models for Internal Dosimetry of the Human Respiratory Tract (Report of the International Commission on Radiological Protection: ICRP: Task Group on Lung Dynamics). Health Phys. 12:173-207.

9. Raabe, O. G. 1982. Deposition and Clearance of Inhaled Aerosols. Mechanism in Respiratory Toxicology, Vol. 1, pp. 27-76. H. Witschi and P. Nettesheim, Eds. CRC Press, Boca Raton, FL.

10. Dolan, D.F., D. B. Kittelson, and K. T. Whitby. 1975. Measurement of Diesel Exhaust Particle Size Distributions. Paper No. 75-WA/APC-5, American Society of Mechanical Engineers: New York, NY.
11. Dolan, D.F., D.B. Kittelson, and D.Y.H. Pui. 1980. Diesel Exhaust Particle Size Distribution Measurement Techniques. SAE Technical Paper Series, No. 800187.

12. Baumgard, K.J. and D.B. Kittelson. 1985. The Influence of a Ceramic Particle Trap on the Size Distribution of Diesel Particles. SAE Technical Paper Series No. 850009, SAE Transactions, 95:56-69.

13. Abdul-Khalek, I.S. and D.B. Kittelson. 1995. Real Time Measurement of Volatile and Solid Exhaust Particles Using a Catalytic Stripper. SAE Technical Paper Series No. 950236.

14. Abdul-Khalek, I. S., D. B. Kittelson, B. R. Graskow, Q. Wei, and F. Brear, 1998a, Diesel Exhaust Particle Size: Measurement Issues and Trends, SAE paper number 98P-353.

15. Kittelson, D. B. 1998. Engines and Nanoparticles: A Review. J. Aerosol Sci. Vol. 29, NO. 5/6, pp. 575588.

16. Bagley, S. T., K.J. Baumgard, L.D. Gratz, J.H. Johnson, and D.G. Leddy. 1996. Characterization of Fuel and Aftertreatment Device Effects on Diesel Emissions. Health Effects Institute Research Report No. 76.

17. Bagan, J., Study of Particle Size Distributions Emitted by a Diesel Engine", SAE Paper No. 1999-011141, 1999.

18. Kruger, M., Luders, H., Luers, B., Kaufmann, R., Koch, W., and Kauffeldt, T. 1997. Influence of Exhaust Gas Aftertreatment on Particulate Characteristics of Vehicle Diesel Engines, Motortechnische Zeitschrift, Vol. 58.

19. Mayer, A., Egli, H., Burtscher, H., Czerwinski, J., and Gehrig, H. 1995. Particle Size Distribution Downstream Traps of Different Design, SAE Technical Paper Series No. 950373.

20. Whitby, K. T., et al. 1975. Characterization of California Aerosols - 1. Size Distributions of Freeway Aerosol. Atmosph. Environ. 9:463-482.

21. Wilson, W. E., et al. 1977. General Motors Sulfate Dispersion Experiment: Summary of EPA Measurements. JAPCA 27(1):46-51.

22. Kittelson, D. B., et al. 1988. Characterization of Diesel Particles in the Atmosphere. Coordinating Research Council AP-2 Project Group Final Report.

23. Harrison, R. M., et al. 1996. Airborne Particulate Matter in the United Kingdom. Third Report of the Quality of Urban Air Review Group, University of Birmingham, Birmingham, UK. 176 pp. 
24. Weingartner, E., et al. 1997(a). Aerosol Emission in a Road Tunnel. Atmosph. Environ. 31(3):451-462

25. Abdul-Khalek, I. S., D. B. Kittelson, and F. Brear. 1998b. Diesel Trap Performance: Particle Size Measurements and Trends. SAE Tech. Pap. Ser. No. 982599.

26. Abdul-Khalek, I.S., D.B. Kittelson, and F. Brear. 1999. Influence of Dilution Conditions on Diesel Exhaust Particle Size Distribution Measurements. SAE Paper No. 1999-01-1142.

27. Khalek, Imad A., D.B. Kittelson, and F. Brear. 2000 Nanoparticle Growth during Dilution and Cooling of Diesel Exhaust: Experimental Investigation and Theoretical Assessment. SAE Paper No. 2000-01-0515
28. http://www.me.umn.edu/divisions/mel/reports/ crcsum1.pdf

29. H. W. Gäggeler et al., The Epiphaniometer, a new device for continuous aerosol monitoring, J. Aerosol Sci. 20, 557-564 (1989).

30. U. Matter et al., Dynamic field measurements of submicron particles from Diesel engines, Environ. Sci. Technol. 33, 1946-1952 (1999).

31. Wei, Qiang, 1999. "Mini-Dilution Tunnel Performance," M.S. Thesis, University of Minnesota. 